\title{
$\mathrm{S}$ 掺杂 Fe-NC 单原子催化剂氧还原机理研究

\author{
鲁效庆* 曹守福魏晓飞李邵仁魏淑贤*
} \\ (中国石油大学 材料科学与工程学院 青岛 266580)
}

\begin{abstract}
摘要 杂原子掺杂的 $\mathrm{Fe}-\mathrm{NC}$ 催化剂在氧还原反应中表现出优异的性能. 本工作采用密度泛函理论研究了 $\mathrm{S}$ 原子掺杂对 $\mathrm{Fe}-\mathrm{NC}$ 单原子催化剂电子结构的调控及促进氧还原反应的作用机理, 分析了硫原子掺杂后 $\mathrm{Fe}-\mathrm{NC}$ 催化剂的稳定构型, S 原子对 $\mathrm{FeN}_{4}$ 活性位点电子结构的调控, 以及氧气的吸附和氧还原反应作用机理. 研究结果表明, 在 $\mathrm{FeN}_{4}$ 活性位点周围 掺杂少量 $\mathrm{S}$ 原子, 可以提高催化剂的稳定性. $\mathrm{S}$ 原子掺杂提高氧还原性能的机理为: (1) $\mathrm{S}$ 原子的掺杂降低了催化剂的带 隙, 提高催化剂导电性, 有利于电催化氧还原反应; (2) S 原子的掺杂可以提高催化剂吸附氧气的能力, 有利于氧还原反 应; (3) 体系中引入四个 $\mathrm{S}$ 原子可以降低氧还原反应的过电位，提高 $\mathrm{FeN}_{4}$ 位点催化氧还原反应的活性. 这项工作可能为 基于碳材料的单原子催化剂上杂原子掺杂的调控提供新的思路.
\end{abstract}

关键词 $\mathrm{Fe}-\mathrm{NC}$ 催化剂; 杂原子掺杂; 氧还原反应; 密度泛函理论

\section{Investigation on Oxygen Reduction Reaction Mechanism on S Doped Fe-NC Isolated Single Atoms Catalyst}

\author{
Lu, Xiaoqing* Cao, Shoufu Wei, Xiaofei Li, Shaoren Wei, Shuxian* \\ (School of Materials Science and Engineering, China University of Petroleum, Qingdao 266580, China)
}

\begin{abstract}
Heteratom-doped Fe-NC catalyst is promising for highly efficiently oxygen reduction reaction (ORR). In this work, density functional theory with the Vienna Ab initio Simulation Package (VASP) has been employed to systematically study the electronic structure regulation mechanism and oxygen reduction promoting mechanism on sulfur atom doped Fe-NC catalyst. The Perdew-Burke-Ernzerhof (PBE) exchange-correlation functional within a generalized gradient approximation (GGA) was used in this work. The computataional hydrogen electron model was used to calculate the changes in Gibbs free energy. To consider the influence of S doping proportion, we build FeNSx models with $1 \sim 4 \mathrm{~S}$ atoms. The thermodynamic stability of catalysts was firstly considered based on formation energy, following by electronic structure analysis through Bader charge analysis and densities of states. Then, the oxygen adsorption ability was considered based on oxygen adsorption configurations and energies analyses. At last, reaction overpotentials were calculated based on computational hydrogen electrode model to study activity of catalytic sites. The results show that the catalyst doped with few sulfur atoms around the active sites of FeN4 could remain stable. Three possible ORR promoting mechanisms of S atoms doping were investigated. Firstly, the doping of sulfur atoms would reduce the band gap of the catalyst, thus improving the conductivity of the catalyst, which is beneficial to electrocatalytic oxygen reduction reactions. Secondly, the doping of a small amount of S atoms can improve the affinity between oxygen and the catalysts, which is also important for oxygen reduction reaction. At last, the introduction of four $\mathrm{S}$ atoms in the system would reduce the overpotential of ORR, thus improving the activity of the active sites to catalyze the oxygen reduction reaction. Our results predict that few $\mathrm{S}$ atoms doping would improve ORR performance of the Fe-NC catalyst through reducing band gap, improving ability to adsorb oxygen, and improving catalytic activity of $\mathrm{FeN}_{4}$ site. This work may give a new insight into regulation rules of heteratom doping on single atom catalysts based on carbon materials.

Keywords Fe-NC catalyst; heteratom doping; oxygen reduction reaction; density functional theory
\end{abstract}

\section{1 引言}

能源可持续发展是当今经济和社会可持续发展的 重要保障, 同时也是科学研究密切关注的热门话题 ${ }^{[1]}$. 新一代能源转换装置及能源储存设备的研究成为迫在 眉睫的任务, 而燃料电池 ${ }^{[2]}$ 和金属空气电池 ${ }^{[3]}$ 等新能源
的开发依赖于氧还原反应的催化剂. 传统的 Pt 基催化 剂在电催化氧还原方面具有较高的催化活性 ${ }^{[4]}$, 但是 $\mathrm{Pt}$ 金属的成本较高, 资源有限, 且长期稳定性差等因素限 制了大规模商业化应用 ${ }^{[5]}$.

非金属催化剂不仅可以降低催化剂的成本，还可以 保持较高的电催化氧还原活性 ${ }^{[6]}$. 在实际的燃料电池测

*E-mail: luxq@upc.edu.cn,wshx@upc.edu.cn

Received June 10, 2020; published July 25, 2020.

Project supported by the Major Scientific and Technological Projects of China National Petroleum Corporation (ZD2019-184-001) and Fundamental Research Funds for the Central Universities (18CX02042A and 18CX05011A).

项目受中石油重大科技项目(ZD2019-184-001)和中央高校自主创新交叉专项(18CX02042A, 18CX05011A)资助. 
试中, 氮掺杂碳材料展示出了比 $\mathrm{Pt}$ 基催化剂更优异的 催化活性和稳定性 ${ }^{[7]}$. 在此基础上, 含氮碳材料表面掺 杂金属单原子(M-NC)可以进一步提高材料的催化活性. 该类催化剂表面的活性位点为 $\mathrm{MN}_{4}$ 位点 ${ }^{[8]}$. 其中, $\mathrm{Fe}-\mathrm{NC}$ 在电催化氧还原方面展现出优异的催化性能 ${ }^{[9]}$. 对催化活性位点结构进行精确调控可以进一步优化其 催化性能. 在 Fe-NC 催化剂表面掺杂杂质原子, 尤其是 $\mathrm{S}$ 原子, 可以调控电子结构, 提高其催化性能 ${ }^{[10]} . \mathrm{S}$ 原子 与 $\mathrm{N}$ 原子相类似, 同为 $\mathrm{p}$ 区元素, 但是两者的电负性和 原子半径都有很大的差异. $\mathrm{S}$ 原子具有相对较大的原子 半径, 所以容易在碳材料表面引入缺陷; 另外, $\mathrm{S}$ 原子的 电负性较低, 可以调节 $\mathrm{FeN}_{4}$ 活性位点的电子结构. 实验 上可以通过高温下热解含 $\mathrm{S}$ 分子、盐等物质在 $\mathrm{N}$ 掺杂碳 材料上引入 $\mathrm{S}$ 原子, 如 $\mathrm{Fe}(\mathrm{SCN})_{3}{ }^{[11]}, \mathrm{CS}\left(\mathrm{NH}_{2}\right)_{2}{ }^{[12]}$, 二硫 代乙酰胺锌 ${ }^{[13]}$ 以及三聚㲵胺-芐基二硫化物树脂 ${ }^{[14]}$; 或 者用等离子处理和化学气相沉积 ${ }^{[15]}$.

提高电催化氧还原反应的催化性能有三个主要因 素: (1)提高催化剂整体导电性; (2)提高活性位点吸附氧 气的能力; (3)降低电催化过电势从而提高催化剂活性. $\mathrm{S}$ 原子掺杂 $\mathrm{Fe}-\mathrm{NC}$ 可以显著提高其电催化氧还原反应的 催化性能, 但是, 目前仍不清楚 S 原子掺杂对 Fe-NC 催 化性能具体的调控机理. 因此, 深入系统地研究 $\mathrm{S}$ 原子 掺杂对催化性能的促进机理十分必要. 本工作采用密度 泛函理论研究了 $\mathrm{S}$ 原子掺杂 $\mathrm{Fe}-\mathrm{NC}$ 的结构和稳定性, 催 化剂的电子结构, 氧气在活性位点的吸附, 以及在活性 位点氧还原反应机理.

\section{2 结果与讨论}

\section{$2.1 \mathrm{FeNS}$ 的结构与稳定性}

大量的研究已经表明, 单原子铁掺杂含氮碳材料的 催化活性中心是 $\mathrm{FeN}_{4}$ 位点, 即铁单原子与四个吡啶氮 配位, 如图 1 所示 ${ }^{[16]}$. 因此, 我们研究的 Fe-NC 催化剂 采用与文献中相同的 $\mathrm{FeN}_{4}$ 模型, 在此基础上研究 $\mathrm{S}$ 原子 掺杂的可能构型. 根据 $\mathrm{Li}$ 等 ${ }^{[10]}$ 的研究, $\mathrm{S}$ 原子和 $\mathrm{N}$ 原子 的比例为 $n(\mathrm{~S}): n(\mathrm{~N})=1: 1$ 时, FeNS 催化剂催化氧还原 反应的活性是最高的, 为了研究 $\mathrm{S}$ 掺杂比例的影响, 我 们考虑了 $1 \sim 4$ 个 $\mathrm{S}$ 原子掺杂的构型, 如图 1 所示, 一个 $\mathrm{S}$ 原子掺杂有两种可能的构型, 分别为 $\mathrm{S}$ 原子与 $\mathrm{N}$ 原子 成键(FeNS1a) 和 $\mathrm{S}$ 原子不与 $\mathrm{N}$ 原子成键(FeNS1b). 形成 能的数值小于零, 表明该结构是热力学稳定的, 形成能 的数值大于零, 表明该结构是不稳定的. 根据形成能计 算(表 1), FeNS1a 的形成能为 $-0.06 \mathrm{eV}, \mathrm{FeNS} 1 \mathrm{~b}$ 的形成 能为 $0.50 \mathrm{eV}$, 结构 FeNS1a 的稳定性优于结构 FeNS1b, 因此 2 4 个 $\mathrm{S}$ 原子掺杂的 FeNS 只考虑 $\mathrm{S}$ 原子与 $\mathrm{N}$ 原 子直接成键结构. 在 FeNS2 结构中, 引入两个 $\mathrm{S}$ 原子的 形成能为 $-0.27 \mathrm{eV}$, 表明其热力学稳定性优于 FeNS1a 结构, 进一步提高 $\mathrm{S}$ 原子掺杂的量, 可以进一步提高催

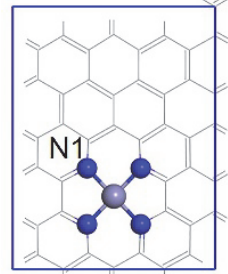

$\mathrm{FeN}_{4} 0.188 \mathrm{~nm}$

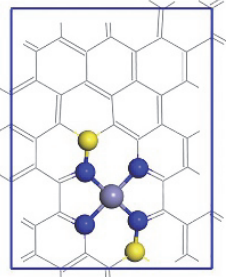

FeNS2 $0.183 \mathrm{~nm}$

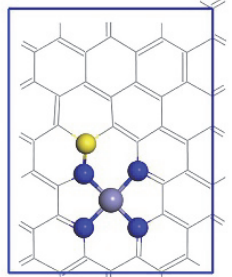

FeNS1a $0.183 \mathrm{~nm}$

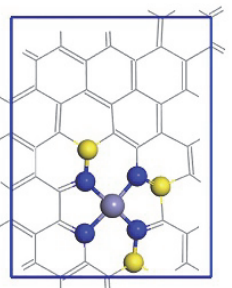

FeNS3 $0.187 \mathrm{~nm}$

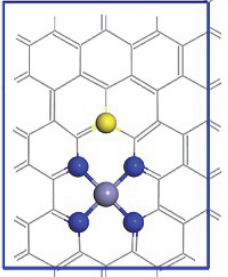

FeNS1b $0.189 \mathrm{~nm}$

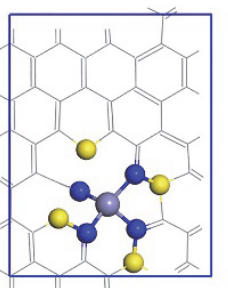

FeNS4 $0.201 \mathrm{~nm}$
图 $1 \mathrm{FeN}_{4}$ 及硫原子掺杂结构示意图

Figure 1 The optimized structures of $\mathrm{FeN}_{4}$ and FeNSx

Blue spheres represent nitrogen atoms, yellow spheres represent sulfur atoms, and gray spheres represent iron atoms, shown here. The number is the length of the Fe-n1 bond

表 1 催化剂结构的形成能和具体计算参数

Table 1 Formation energy of catalysts and specific calculation parameters

\begin{tabular}{lccccr}
\hline & $E(\mathrm{FeNSx}) / \mathrm{eV}$ & $\mu(\mathrm{C}) / \mathrm{eV}$ & $\mu(\mathrm{S}) / \mathrm{eV}$ & $E\left(\mathrm{FeN}_{4}\right) / \mathrm{eV}$ & $E_{\mathrm{f}} / \mathrm{eV}$ \\
\hline FeNS1a & -421.13 & -9.26 & -0.94 & -429.40 & -0.06 \\
FeNS1b & -420.58 & -9.26 & -0.94 & -429.40 & 0.50 \\
FeNS2 & -413.02 & -9.26 & -0.94 & -429.40 & -0.27 \\
FeNS3 & -404.91 & -9.26 & -0.94 & -429.40 & -0.49 \\
FeNS4 & -397.82 & -9.26 & -0.94 & -429.40 & -1.74 \\
\hline
\end{tabular}

化剂结构的稳定性, 在 $\mathrm{FeN}_{4}$ 位点引入四个 $\mathrm{S}$ 原子掺杂 时, 结构的形成能为 $-1.74 \mathrm{eV}$. 形成能的计算结果表明, 在 $\mathrm{FeN}_{4}$ 位点周围引入 $\mathrm{S}$ 原子掺杂可以提高催化剂结构 的稳定性. 通过计算 $\mathrm{Fe}-\mathrm{N}$ 键长发现, 硫原子掺杂对 $\mathrm{Fe}$ $-\mathrm{N}$ 键长有较大影响. $\mathrm{FeN}_{4}$ 结构中 $\mathrm{Fe}-\mathrm{N}$ 键的键长为 $0.188 \mathrm{~nm}$, 而 $\mathrm{FeNS} 1 \mathrm{a}$ 和 $\mathrm{FeNS} 1 \mathrm{~b}$ 中 $\mathrm{Fe}-\mathrm{N}$ 的键长分别为 $0.183 \mathrm{~nm}$ 和 $0.189 \mathrm{~nm}, \mathrm{FeNS} 2$ 结构中 $\mathrm{Fe}-\mathrm{N}$ 的键长为 $0.183 \mathrm{~nm}$. FeNS1a 和 FeNS2 构型中 $\mathrm{Fe}-\mathrm{N}$ 键长明显缩 短, 此时所有的原子仍然在同一平面内. 在 FeNS3 结构 中, $\mathrm{Fe}-\mathrm{N}$ 键长为 $0.187 \mathrm{~nm}, \mathrm{FeNS} 4$ 结构中, $\mathrm{Fe}-\mathrm{N}$ 键长 为 $0.201 \mathrm{~nm}$, 此时, 由于 $\mathrm{S}$ 原子的原子半径大于氮原子, 所以过多 $\mathrm{S}$ 原子的掺杂导致空缺位的出现, 此时, 其中 一个 $\mathrm{S}$ 原子与 $\mathrm{N}$ 原子断键.

\subsection{Fe-NS 催化剂电子结构的研究}

为了研究 $\mathrm{S}$ 原子掺杂对 $\mathrm{FeN}_{4}$ 催化剂电子结构的影 响, 我们计算了五个研究结构的巴德电荷. 巴德电荷分 析如表 2 所示. $\mathrm{FeN}_{4}$ 结构中铁原子的电子转移数为 1.08 
$|\mathrm{e}|$, 引入一个 $\mathrm{S}$ 原子后 $\mathrm{Fe}$ 原子的电子转移数变为 1.05 $|\mathrm{e}|$. 引入两个 $\mathrm{S}$ 原子后, $\mathrm{Fe}$ 原子向周围转移 $1.02|\mathrm{e}|$ 的电 子数, 在 FeNS3 结构中, $\mathrm{Fe}$ 原子向周围转移的电子数为 $0.90|\mathrm{e}|$, 但是在 FeNS4 结构中, Fe 原子失去的电子数为 $1.14 \mathrm{e} \mid$, 高于在其他结构中的电子转移数, 因而, 在 FeNS4 结构中, $\mathrm{Fe}$ 的价态高于 $\mathrm{FeN}_{4}$ 中 $\mathrm{Fe}$ 的价态. 接下 来, 我们进一步利用电子态密度分析 $\mathrm{Fe}$ 原子与周围配 位环境的成键情况, 如图 2 所示. 因为在实际成键过程 中起主要作用的是最外层的电子, 所以对于 $\mathrm{Fe}$ 原子而 言，只考虑其 $3 \mathrm{~d}$ 轨道，对于 $\mathrm{N}$ 原子和 $\mathrm{S}$ 原子而言，只考 虑其 $2 \mathrm{p}$ 轨道. 结果表明, $\mathrm{FeN}_{4}$ 催化剂表面 $\mathrm{Fe}$ 原子 $3 \mathrm{~d}$ 轨 道的电子密度较大, 且 $\mathrm{Fe}$ 原子的 $3 \mathrm{~d}$ 轨道发生明显的电 子自旋现象, 由于轨道强相互作用, 其自旋向上的轨道 向费米能级以下偏移了大约 $2.0 \mathrm{eV}$, 导致在费米能级附 近只有自旋向下的轨道, 而且此自旋向下的轨道在费米 能级处的带隙为 $0.907 \mathrm{eV}$. 引入一个 $\mathrm{S}$ 原子掺杂后, 催 化剂的带隙明显降低, 在 FeNS1a 中, 带隙为 $0.449 \mathrm{eV}$. 引入 $\mathrm{S}$ 原子的 $2 \mathrm{p}$ 轨道, 没有引起 $\mathrm{Fe}$ 原子的 $3 \mathrm{~d}$ 轨道杂化, 但是会降低催化剂能带的带隙. 引入更多 $\mathrm{S}$ 原子时, 能 带结构表现出同样的规律, FeNS2 催化剂的带隙为 0.351 $\mathrm{eV}, \mathrm{FeNS} 3$ 催化剂的带隙为 $0.254 \mathrm{eV}$, FeNS4 催化剂的带 隙为 $0.121 \mathrm{eV}$. 电子态密度及能带分析表明, $\mathrm{FeN}_{4}$ 催化 剂掺杂 $\mathrm{S}$ 原子的比例越高, 催化剂电子结构的带隙变得
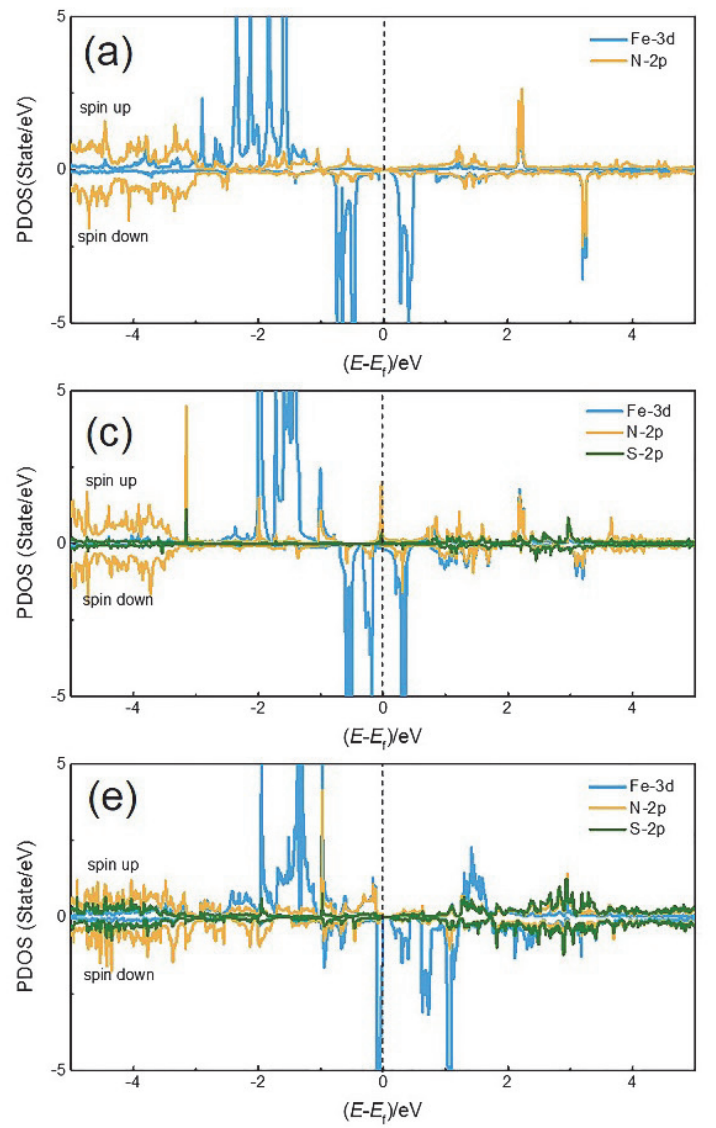

越窄从而增强催化剂导电能力, 在电催化的过程中更有 利于电子的转移, 因此, 在 $\mathrm{FeN}_{4}$ 催化剂中引入 $\mathrm{S}$ 原子可 以显著提高催化剂的电子转移速率.

\section{3 氧气的吸附机理}

催化剂对氧气的吸附能力对催化剂整体氧还原性 能至关重要. 氧气在不同催化剂表面的吸附构型和吸附 能如图 3 所示. 我们发现了两种氧气吸附构型, 分别命 名为 side-on 和 end-on 吸附构型. 在 side-on 吸附构型中, $\mathrm{O}_{2}$ 分子中的两个氧原子都与基底成键，在 end-on 吸附 构型中, 氧气分子坚直吸附在催化剂表面, 其中的一个 氧原子与催化剂相连. 在 $\mathrm{FeN}_{4}$ 表面, 氧气在催化剂表面 倾向于以 end-on 构型吸附, 吸附能为 $-2.03 \mathrm{eV}$,

表 2 催化剂的巴德电荷分析

Table 2 Bader charge analyses of catalysts

\begin{tabular}{cccccc}
\hline & $\mathrm{FeN}_{4}(|\mathrm{e}|)$ & $\mathrm{FeNS1a}(|\mathrm{e}|)$ & $\mathrm{FeNS} 2(|\mathrm{e}|)$ & $\mathrm{FeNS} 3(|\mathrm{e}|)$ & $\mathrm{FeNS4}(|\mathrm{e}|)$ \\
\hline $\mathrm{Fe}$ & -1.08 & -1.05 & -1.02 & -0.90 & -1.14 \\
$\mathrm{~N}$ & 1.11 & 1.17 & 1.17 & 1.18 & 1.21 \\
$\mathrm{~S} 1$ & & -0.75 & -0.77 & -0.78 & -0.15 \\
$\mathrm{~S} 2$ & & & -0.81 & -0.70 & -0.72 \\
$\mathrm{~S} 3$ & & & & -0.95 & -0.83 \\
$\mathrm{~S} 4$ & & & & & -0.42 \\
\hline
\end{tabular}
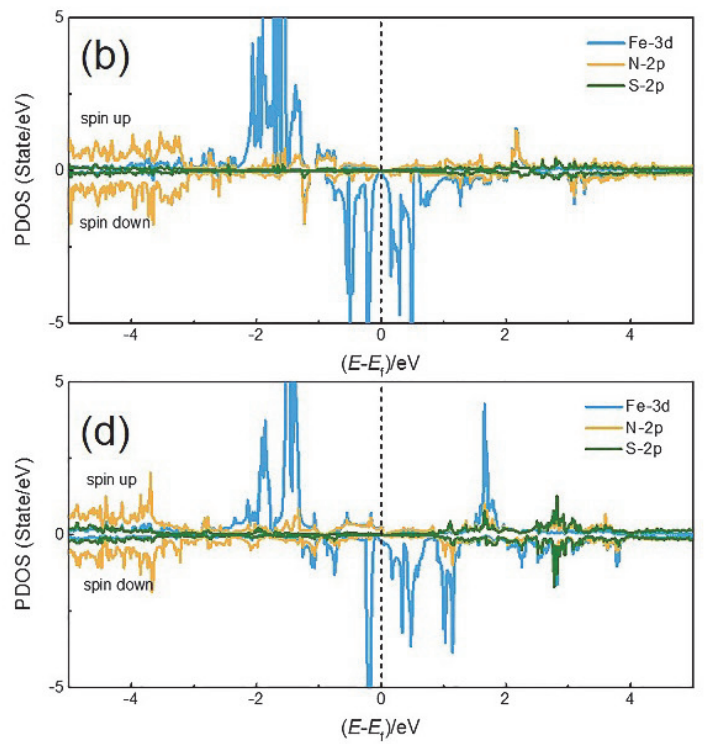

图 2 态密度图(a) $\mathrm{FeN}_{4}$, (b) FeNS1a, (c) FeNS1b, (d) FeNS2 和(e) FeNS3

Figure 2 Densities of states of (a) $\mathrm{FeN}_{4}$, (b) FeNS1a, (c) FeNS1b, (d) FeNS2 and (e) FeNS3 
其 side-on 吸附构型的吸附能为 $-1.93 \mathrm{eV}$. 相比于在 $\mathrm{FeN}_{4}$ 催化剂表面的吸附, 氧气在 FeNS1 催化剂表面的 吸附更稳定, 表明在 $\mathrm{FeN}_{4}$ 催化剂表面引入一个 $\mathrm{S}$ 原子掺 杂可以提高氧气的吸附能力, 从而提高氧气分子的活化 性能. 计算结果表明, 一个 $\mathrm{S}$ 原子掺杂的催化剂中, 氧 气倾向于以 side-on 吸附构型吸附在催化剂表面, 在 FeNS1a 催化剂上, 其吸附能为 $-2.18 \mathrm{eV}$, 继续增加 $\mathrm{S}$ 原 子掺杂的比例, 相比于掺杂一个 $\mathrm{S}$ 原子的催化剂, 氧气 的吸附能降低. 在 FeNS2 催化剂表面, 氧气倾向于 side-on 吸附构型, 其吸附能为 $-2.16 \mathrm{eV}$, 氧气以 end-on 构型吸附的吸附能为 $-2.06 \mathrm{eV}$. 在 FeNS3 催化剂表面, 氧气只有 end-on 吸附构型, 其吸附能为 $-1.97 \mathrm{eV}$, 吸 附能低于氧气在 $\mathrm{FeN}_{4}$ 催化剂表面的吸附能. 在 FeNS4 催化剂表面, 氧气的吸附构型为 end-on, 吸附能为 $-1.97 \mathrm{eV}$. 氧气在 $\mathrm{S}$ 原子掺杂 $\mathrm{Fe}-\mathrm{NC}$ 催化剂表面的吸附 研究表明, 引入少量的 $\mathrm{S}$ 原子掺杂可以提高催化剂吸附 氧气的能力, 提高 $\mathrm{S}$ 原子掺杂比例, 将降低其对氧气的 吸附能力.

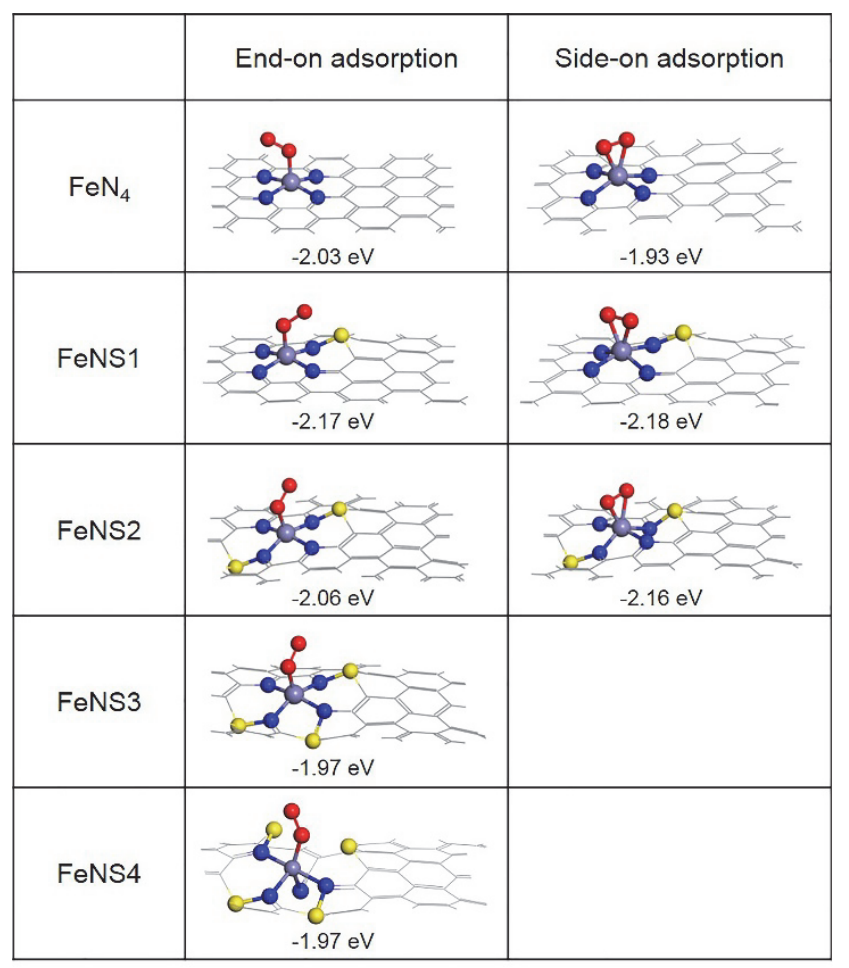

图 3 氧气可能吸附构型

Figure 3 The optimized adsorption configurations of oxygen

\section{4 氧还原反应机理研究}

我们对催化剂活性位点的氧还原催化活性进行具 体的研究. 根据 $\mathrm{Li}$ 等 ${ }^{[10]}$ 的研究, 氧气在 Fe-NS 催化剂表 面的还原反应通过四个电子反应步骤生成水. 因此, 我 们主要针对这一机理进行研究. 在反应自由能的计算过 程中, 因为 VASP 计算双原子气体分子的振动频率不够 准确, 因此我们采用 Norskov 等 ${ }^{[17]}$ 应用的方法, 用氧还
原反应的标准电极电势减去其他三项反应步骤的自由 能, 得到第一步反应的反应自由能: $\mathrm{O}_{2}+*+\left(\mathrm{H}^{+}+\right.$ $\left.\mathrm{e}^{-}\right) \rightarrow{ }^{*} \mathrm{OOH}$. 氧还原反应的平衡电极电势为 $4.92 \mathrm{~V}$, 为 了显著表示氧还原反应的过电位，我们计算了 $U=1.23$ $\mathrm{V}$ 时的反应自由能. 氧还原反应过程中涉及到的反应中 间体有 $* \mathrm{OOH}, * \mathrm{O}$ 和 $* \mathrm{OH}(*$ 表示中间体在催化剂表面吸 附). 氧还原反应自由能图如图 4, 反应过程中涉及到的 反应中间体吸附构型如图 5 , 具体的反应自由能能量如 表 3 所示. 在 $\mathrm{FeN}_{4}$ 催化剂表面, 氧气以 end-on 构型稳定 吸附在铁原子的顶位上, 溶液中一个质子-电子对转移 到氧气分子上, 质子与远端的氧原子连接, 形成 $\mathrm{OOH}^{*}$ 中间体, 该步反应的自由能为 $-0.27 \mathrm{eV}$, 是自发进行的. 接下来 $\mathrm{OOH}^{*}$ 中间体接收一对质子-电子, 生成一个水 分子和吸附的氧原子 $\mathrm{O}^{*}$, 该步反应的反应自由能为 $-0.91 \mathrm{eV}$, 同样是自发进行的, 表明一旦氧气吸附在 $\mathrm{FeN}_{4}$ 表面, 就会自发生成 $\mathrm{O}^{*}$ 和一个水分子. 接下来 $\mathrm{O}^{*}$ 经过质子-电子反应生成 $\mathrm{OH}^{*}$, 需要克服 $0.48 \mathrm{eV}$ 的能 量. 最后, 一个质子转移到 $\mathrm{O}$ 原子上生成一个水分子, 需要克服 $0.70 \mathrm{eV}$ 的能量, 最后一步反应是氧还原反应 的电势控制步骤, 表明在 $\mathrm{FeN}_{4}$ 催化剂上氧还原反应的 过电位是 $0.70 \mathrm{~V}$. 在 FeNS1a 和活性位点, 氧还原反应的 电势控制步骤同样是最后一步，过电位为 $0.79 \mathrm{~V}$, 表明 引入一个 $\mathrm{S}$ 原子之后不仅没有降低氧还原反应的过电 位, 反而使得过电位增加. 在 FeNS2 催化剂表面, 由于 $\mathrm{OOH}^{*}$ 的吸附能力较弱, 初步反应需要较大的能量, 为 $0.21 \mathrm{eV}$, 氧还原反应的电势控制步骤是 $\mathrm{OH}^{*}+\left(\mathrm{H}^{+}+\mathrm{e}^{-}\right)$ $\rightarrow *+\mathrm{H}_{2} \mathrm{O}(\mathrm{l})$, 过电位是 $0.82 \mathrm{~V}$. 在 FeNS3 催化剂表面, 电势控制步骤与 $\mathrm{FeN}_{4}$ 表面一样, 过电位是 $0.77 \mathrm{~V}$. 在 FeNS4 催化剂表面, 电势控制步骤同样为最后一步, 过 电位是 $0.68 \mathrm{~V}$, 在五种研究的催化剂中, 其过电位是最 低的. 对比巴德电荷分析, FeNS4 催化剂中, Fe 的价态是 最高的, 表明 $\mathrm{Fe}$ 较高的氧化态可以降低反应中间体 $* \mathrm{OH}$ 的吸附.

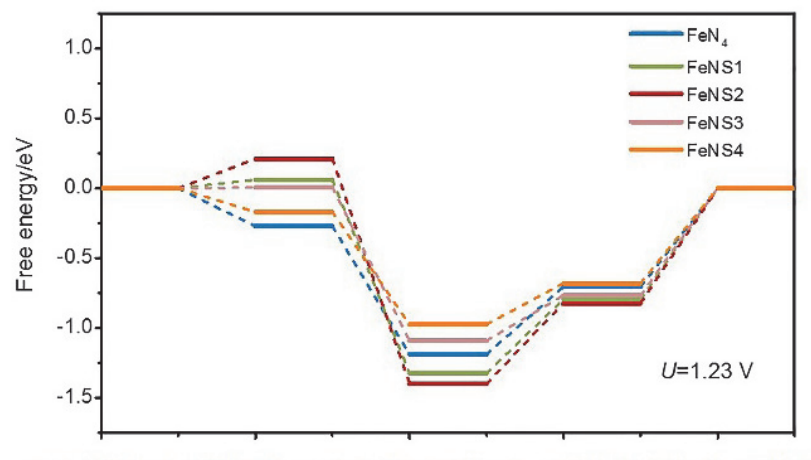

${ }^{*}+\mathrm{O}_{2}+4\left(\mathrm{H}^{+}+\mathrm{e}^{-}\right) \quad{ }^{*} \mathrm{OOH}+3\left(\mathrm{H}^{+}+\mathrm{e}^{-}\right) \quad{ }^{*} \mathrm{O}+\mathrm{H}_{2} \mathrm{O}+2\left(\mathrm{H}^{+}+\mathrm{e}^{-}\right) \quad{ }^{*} \mathrm{OH}+\mathrm{H}_{2} \mathrm{O}+\left(\mathrm{H}^{+}+\mathrm{e}^{-}\right) \quad{ }^{*}+2 \mathrm{H}_{2} \mathrm{O}$

图 4 氧还原反应自由能图及反应中间体吸附结构

Figure 4 Reaction energy diagram for the catalysts during ORR and optimized adsorption configurations of reaction intermediates 


\begin{tabular}{|c|c|c|c|}
\hline & $\mathrm{OOH}^{*}$ & $0^{*}$ & $\mathrm{OH}^{*}$ \\
\hline $\mathrm{FeN}_{4}$ & 20 & 20 & 200 \\
\hline FeNS1a & क्ष० & 20 & 305 \\
\hline FeNS2 & & 203 & \\
\hline FeNS3 & & 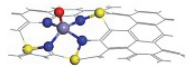 & 를 \\
\hline FeNS4 & 20 & 3 & 3 \\
\hline
\end{tabular}

图 5 氧还原反应中间体吸附结构

Figure 5 Optimized adsorption configurations of reaction intermediates during ORR

表 3 氧还原反应能量

Table 3 Reaction energies of oxygen reduction reaction

\begin{tabular}{lrrcc}
\hline & $\Delta G_{1} / \mathrm{eV}$ & $\Delta G_{2} / \mathrm{eV}$ & $\Delta G_{3} / \mathrm{eV}$ & $\Delta G_{4} / \mathrm{eV}$ \\
\hline FeN $_{4}$ & -0.27 & -0.91 & 0.48 & 0.70 \\
FeNS1a & 0.06 & -1.38 & 0.52 & 0.79 \\
FeNS2 & 0.21 & -1.61 & 0.57 & 0.82 \\
FeNS3 & 0.01 & -1.09 & 0.32 & 0.76 \\
FeNS4 & -0.17 & -0.80 & 0.29 & 0.68 \\
\hline
\end{tabular}

\section{3 结论}

本工作基于密度泛函理论, 对在 $\mathrm{FeN}_{4}$ 催化剂表面 引入硫原子掺杂的电子调控机理进行了计算研究, 研究 结果如下.

(1) 在 $\mathrm{FeN}_{4}$ 催化剂表面掺杂 $\mathrm{S}$ 原子可以提高催化剂 的稳定性, 掺杂 $\mathrm{S}$ 原子的比例越高, 催化剂越稳定.

(2) $\mathrm{S}$ 原子掺杂可以降低 $\mathrm{FeN}_{4}$ 催化剂的带隙, 提高 导电性, 有利于氧还原反应过程中电子的转移, 有利于 电催化反应的进行. 在 FeN4 活性位点周围引入一个 $\mathrm{S}$ 原子时, 其带隙由 $0.907 \mathrm{eV}$ 降低为 $0.449 \mathrm{eV}$, 导电性质 明显改善.

（3）相比于 $\mathrm{FeN}_{4}$ 结构, 在 FeNS1 的两个结构中, 氧 气的吸附能力明显提高, 在此基础上增加 $\mathrm{S}$ 原子掺杂的 比例, 其氧气吸附能力反而下降, 因此, 在 $\mathrm{FeN}_{4}$ 中引入 少量 $\mathrm{S}$ 原子掺杂, 可以提高催化剂对氧气的吸附能力.

(4) 氧还原反应机理研究表明, 在 $\mathrm{FeN}_{4}$ 催化剂表面 引入四个 $\mathrm{S}$ 原子形成 FeNS4 位点可以降低氧还原过电 势, 提高催化位点的活性.

\section{4 计算方法}

所有计算均采用自旋极化密度泛函理论(DFT)和 Vienna 从头算模拟包(VASP)进行 ${ }^{[18]}$. 本研究使用广义
梯度近似(GGA)下的 Perdew-Burke-Ernzerhof (PBE) 交 换相关函数 ${ }^{[19]}$. 为了描述离子核, 应用了投影缀加平面 波(PAW) 赝势 ${ }^{[18]}$. 几何优化的 $k$ 点设置为 $5 \times 5 \times 1$, DOS 计算的 $k$ 点设置为 $20 \times 20 \times 1$. 截断能量采用 $500 \mathrm{eV}$. 电 子能量和力的收玫标准分别为 $10^{-5} \mathrm{eV}$ 和 $0.01 \mathrm{eV} / \AA$. 在 采用 Grimme (DFT-D3)方法时考虑到了范德瓦尔斯相互 作用. 在我们的计算中, 采用 VASP 隐式溶剂模型考虑 水的影响 ${ }^{[20]}$ 以模拟真实的电催化环境.

催化剂的热力学稳定性通过形成能 $\left(E_{\mathrm{f}}\right)$ 计算:

$$
E_{\mathrm{f}}=E(\mathrm{FeNSx})+m \mu(\mathrm{C})-n \mu(\mathrm{S})-E\left(\mathrm{FeN}_{4}\right)
$$

其中 $E($ total $)$ 表示 $\mathrm{S}$ 原子掺杂后催化剂的能量, $E$ (graphite)表示完整碳材料的能量, $\mu(\mathrm{C}) 、 \mu(\mathrm{S}) 、 \mu(\mathrm{Fe})$ 分 别表示 $\mathrm{C}$ 原子、 $\mathrm{S}$ 原子和 $\mathrm{Fe}$ 原子的化学势, 碳原子的化 学势通过完整碳材料的能量除去 $\mathrm{C}$ 原子的数目得到, $m$ 表示得到目标催化剂需要在碳材料表面去掉的碳原子 数目, $n$ 表示催化剂中硫原子的数目, 通过以上公式可 以得到催化剂结构的稳定性, 若催化剂的形成能小于 零, 则表明催化剂的热力学稳定性.

氧气和反应中间体的吸附能 $\left(E_{\mathrm{ads}}\right)$ 计算公式如下:

$$
E_{\mathrm{ads}}=E_{\mathrm{M} / \mathrm{s}}-E_{\mathrm{S}}-E_{\mathrm{M}}
$$

其中 $E_{\mathrm{M} / \mathrm{S}} 、 E_{\mathrm{S}}$ 和 $E_{\mathrm{M}}$ 分别代表吸附后系统的总能量、吸 附前催化剂的能量和吸附物的能量 ${ }^{[21]}$.

计算氢电极(CHE)模型是用来计算吉布斯自由能的 变化 $(\Delta G)$. 在 $\mathrm{CHE}$ 模型中, 所有 $\mathrm{pH}$ 值下, 下列反应在 0 $\mathrm{V}$ 与可逆氢电极平衡

$$
\mathrm{H}^{+} \text {(aq.) }+\mathrm{e}^{-} \leftrightharpoons 1 / 2 \mathrm{H}_{2}(\mathrm{~g})
$$

因此, 在计算其自由能时, 基本步 ${ }^{*} \mathrm{~A}+\mathrm{H}^{+}+\mathrm{e}^{-}$ $\rightarrow{ }^{*} \mathrm{AH}$ 可以被 ${ }^{*} \mathrm{~A}+1 / 2 \mathrm{H}_{2}(\mathrm{~g}) \rightarrow{ }^{*} \mathrm{AH}$ 所代替. 每一状态的 自由能 $(G)$ 如公式(4)所示:

$$
G=E+\mathrm{ZPE}+\int C_{\mathrm{p}} \mathrm{d} T-T S+G_{\mathrm{pH}}
$$

其中, $T$ 是温度, $E$ 是电子能量. $Z \mathrm{PE} 、 \int C_{\mathrm{P}} \mathrm{d} T 、 T S$ 分别为 分子振动贡献的零点能量、从 0 到 $T \mathrm{~K}$ 的焓变以及 $298.15 \mathrm{~K}$ 处的熵修正, 它们是在振动频率分析的基础上 得到的. $G_{\mathrm{pH}}$ 为 $\mathrm{pH}$ 的自由能修正量, 可由 $G_{\mathrm{pH}}=k_{\mathrm{B}} T$ $\ln 10^{*} \mathrm{pH}$ 计算, 本工作假设 $\mathrm{pH}$ 值为 $0^{[22]}$. 利用 $G(\mathrm{U})=$ $G(0 \mathrm{~V})-n e U$ 来计算与应用电势 $(U)$ 有关的自由能, 本工 作中计算了 $U=1.23 \mathrm{~V}$ 时的反应自由能，过电位定义为 最大的反应自由能变, 即:

$$
\eta=\Delta G_{\max }\left(G_{1}, G_{2}, G_{3}, G_{4}\right) / n e
$$

其中, $n$ 为反应过程中转移的电子数.

\section{References}

[1] Birdja, Y. Y.; Pérez-Gallent, E.; Figueiredo, M. C.; Gttle, A. J.; Koper, M. T. M. Nature Energy 2019, 732.

[2] Zhao, W. C; Xu, X.; Bai, H.; Zhang, J.; Lu, S.; Xiang, Y. Acta Chim. Sinica 2020, 78, 69 (in Chinese). (赵韦辰, 徐金金, 白慧娟, 张劲, 卢善富, 相艳, 化学学报, 2020, 78, 69.)

[3] Suntivich, J.; Gasteiger, H. A.; Yabuuchi, N.; Nakanishi, H.; Goodenough, J. B.; Shao-Horn, Y. Nat. Chem. 2011, 3, 546.

[4] Li, J.; Feng, X.; Wei, Z. D. J. Electrochem. 2018, 24, 22 (in Chinese). (李静, 冯欣, 魏子栋, 电化学, 2018, 24, 22.) 
[5] Wang, Y. J.; Zhao, N.; Fang, B.; Li, H.; Bi, X. T.; Wang, H. Chem. Rev. 2015, 115, 3433.

[6] Dai, L.; Xue, Y.; Qu, L.; Choi, H. J.; Baek, J. B. Chem. Rev. 2015, 115,4823 .

[7] Strickland, K.; Miner, E.; Jia, Q.; Tylus, U.; Ramaswamy, N.; Liang, W.; Sougrati, M. T.; Jaouen, F.; Mukerjee, S. Nat. Chem. 2015, 6, 7343.

[8] (a) Huang, W. J.; Zhang, H. Y.; Hu, S.-Z.; Niu, D. F.; Zhang, X.-S. Acta Chim. Sinica 2018, 76, 723 (in Chinese). (黄文姣, 张浩宇，胡 硕真, 钮东方, 张新胜, 化学学报, 2018, 76, 723); (b) Zhang, Z. Q.; Ge, C. X.; Chen, Y. G.; Wu, Q.; Hu, Z. Acta Chim. Sinica 2019, 77,60 (in Chinese). (张志琦, 葛承宣, 陈玉刚, 吴强, 胡征, 化学 学报, 2019, 77, 60); (c) Wang, Y. L.; Wang, M. J.; Li, J.; Wei, Z. D. Acta Chim. Sinica 2019, 77, 84 (in Chinese). (王艺霖, 王敏杰, 李 静, 魏子栋, 化学学报, 2019, 77, 84).

[9] Chang, Z. W.; Meng, F. L.; Zhong, H. X.; Zhang, X. B. Chin. J. Chem. 2018, 36, 287.

[10] Li, Q.; Chen, W.; Xiao, H.; Gong, Y.; Li, Z.; Zheng, L.; Zheng, X.; Yan, W.; Cheong, W. C.; Shen, R.; Fu, N.; Gu, L.; Zhuang, Z.; Chen, C.; Wang, D.; Peng, Q.; Li, J.; Li, Y. Adv. Mater. 2018, 30, e1800588.

[11] Chen, P.; Zhou, T.; Xing, L.; Xu, K.; Tong, Y.; Xie, H.; Zhang, L.; Yan, W.; Chu, W.; Wu, C. Angew. Chem., Int. Ed. 2017, 129, 625.

[12] Hu, K.; Tao, L.; Liu, D.; Huo, J.; Wang, S. ACS Appl. Mater. Interfaces 2016, 8, 30 .

[13] Naveen, M. H.; Shim, K.; Hossain, M. S. A.; Kim, J. H.; Shim, Y. B. Adv. Energy Mater. 2017, 7, 1602002.
[14] Ji, L.; Yan, J.; Jaroniec, M.; Shi, Z. Q. Angew. Chem., Int. Ed. 2012, $51,11808$.

[15] Shen, H.; Gracia-Espino, E.; Ma, J.; Zang, K.; Luo, J.; Wang, L.; Gao, S.; Mamat, X.; Hu, G.; Wagberg, T. Angew. Chem., Int. Ed. 2017, 129, 13988

[16] (a) Lei, C.; Chen, H.; Cao, J.; Yang, J.; Qiu, M.; Xia, Y.; Yuan, C.; Yang, B.; Li, Z.; Zhang, X.; Lei, L.; Abbott, J.; Zhong, Y.; Xia, X.; Wu, G.; He, Q.; Hou, Y. Adv. Energy Mater. 2018, 8, 1801912; (b) Lin, Y.; Liu, P.; Velasco, E.; Yao, G.; Tian, Z.; Zhang, L.; Chen, L. Adv. Mater. 2019, 31, e1808193; (c) Mun, Y.; Lee, S.; Kim, K.; Kim, S.; Lee, S.; Han, J. W.; Lee, J. J. Am. Chem. Soc. 2019, 141, 6254; (d) Wagner, S.; Auerbach, H.; Tait, C. E.; Martinaiou, I.; Kumar, S. C. N.; Kubel, C.; Sergeev, I.; Wille, H. C.; Behrends, J.; Wolny, J. A.; Schunemann, V.; Kramm, U. I. Angew. Chem., Int. Ed. 2019, 58 , 10486.

[17] Norskov, J. K.; Rossmeisl, J.; Logadottir, A.; Lindqvist, L.; Kitchin, J. R.; Bligaard, T.; Jónsson, H. J. Phys. Chem. B 2004, 108, 17886.

[18] Kresse, G.; Furthmuller, J. Phys. Rev. B 1996, 54, 11169.

[19] Perdew, J. P.; Burke, K.; Ernzerhof, M. Phys. Rev. Lett. 1996, 77, 3865.

[20] Mathew, K.; Sundararaman, R.; Letchworth-Weaver, K.; Arias, T. A.; Hennig, R. G. J. Chem. Phys. 2014, 140, 084106.

[21] Guo, C.; Wei, S.; Zhou, S.; Zhang, T.; Wang, Z.; Ng, S. P.; Lu, X.; Wu, C. M. L.; Guo, W. J. ACS Appl. Mater. Interfaces 2017, 9, 26107.

[22] Chen, Z.; Zhao, J.; Cabrera, C. R.; Chen, Z. F. Small Methods 2019, 3,1800368 .

(Cheng, B.) 